\title{
The efficacy of novel anatomical sites for the assessment of muscle oxygenation during central hypovolemia
}

\author{
Justin D Sprick ${ }^{1}$, Babs R Soller ${ }^{2}$ and Caroline A Rickards ${ }^{\mathbf{1}}$ \\ ${ }^{1}$ Institute for Cardiovascular and Metabolic Diseases, University of North Texas Health Science Center, Fort Worth, TX 76107, USA; \\ ${ }^{2}$ Sotera Wireless, San Diego, CA 92121, USA \\ Corresponding author: Caroline A Rickards. Email: caroline.rickards@unthsc.edu
}

\begin{abstract}
Muscle tissue oxygenation $\left(\mathrm{SmO}_{2}\right)$ can track central blood volume loss associated with hemorrhage. Traditional peripheral measurement sites (e.g., forearm) may not be practical due to excessive movement or injury (e.g., amputation). The aim of this study was to evaluate the efficacy of three novel anatomical sites for the assessment of $\mathrm{SmO}_{2}$ under progressive central hypovolemia. 10 male volunteers were exposed to stepwise prone lower body negative pressure to decrease central blood volume, while $\mathrm{SmO}_{2}$ was assessed at four sites - the traditional site of the flexor carpi ulnaris (ARM), and three novel sites not previously investigated during lower body negative pressure, the deltoid, latissimus dorsi, and trapezius. $\mathrm{SmO}_{2}$ at the novel sites was compared to the ARM sensor and to stroke volume responses. A reduction in $\mathrm{SmO}_{2}$ was detected by the ARM sensor at the first level of lower body negative pressure $(-15 \mathrm{mmHg} ; P=0.007)$, and at -30 (the deltoid), -45 (latissimus dorsi), and $-60 \mathrm{mmHg}$ lower body negative pressure (trapezius) at the novel sites $(P \leq 0.04)$. $\mathrm{SmO}_{2}$ responses at all novel sites were correlated with responses at the ARM $(R \geq 0.89)$, and tracked the reduction in stroke volume $(R \geq 0.87)$; the latissimus dorsi site exhibited the strongest linear correlations $(\mathrm{R} \geq 0.96)$. Of the novel sensor sites, the latissimus dorsi exhibited the strongest linear associations with $\mathrm{SmO}_{2}$ at the $\mathrm{ARM}$, and with reductions in central blood volume. These findings have important implications for detection of hemorrhage in austere environments (e.g., combat) when use of a peripheral sensor may not be ideal, and may facilitate incorporation of these sensors into uniforms.
\end{abstract}

Keywords: Trauma, near infrared spectroscopy, physiologic monitoring, tissue oxygen saturation, hypovolemia

Experimental Biology and Medicine 2016; 241: 2007-2013. DOI: 10.1177/1535370216660213

\section{Introduction}

Early and accurate detection of hemorrhagic injury is vital for initiating appropriate interventions in order to improve the chances of survival. Standard vital signs for detection of hemorrhage, such as arterial pressure, arterial oxygen saturation, and radial pulse character, are regulated by reflex compensatory mechanisms, so only begin to change with substantial loss of blood volume. ${ }^{1-3}$ Furthermore, some metrics, such as heart rate (HR), lack specificity to hemorrhage; HR may increase very early due to an underlying hemorrhagic injury, but also due to other stimuli associated with trauma such as pain, anxiety, dehydration, heat or cold stress, physical activity/ movement, and ingestion of exogenous substances. Moreover, in many instances HR will actually decrease with traumatic injury. ${ }^{2,4,5}$ Consequently, by the time these parameters change as a result of injury, it may be too late to effectively intervene and provide potentially lifesaving treatments. For these reasons, research efforts have focused on identifying early and accurate indicators of physiological deterioration.

A number of recent studies have highlighted the potential of measuring muscle tissue oxygen $\left(\mathrm{SmO}_{2}\right)$ as an early marker of central hypovolemia. ${ }^{6-11}$ These studies utilized near infra-red spectroscopy (NIRS) to non-invasively assess oxygen saturation in the flexor carpi ulnaris muscle of the forearm ${ }^{6,8-10,12}$ and the thenar muscles of the hand ${ }^{6,8}$ during progressive reductions in central blood volume elicited via application of lower body negative pressure (LBNP). Forearm $\mathrm{SmO}_{2}$ decreased early during LBNP when stroke volume (SV) was reduced by approximately $7 \%$ from baseline. ${ }^{9}$ By comparison, standard vital signs such as arterial pressure, $\mathrm{HR}$, and arterial oxygen saturation did not change from baseline until at least a $\sim 52 \%$ reduction in $\mathrm{SV}^{9} \mathrm{SmO}_{2}$ continued to fall with progressive application of LBNP until presyncope, with a strong positive linear relationship with SV $\left(R^{2}=0.96\right)$, and an inversely linear relationship with total peripheral resistance (TPR) $\left(R^{2}=0.94\right) .{ }^{9}$ 
A recent study also examined the utility of $\mathrm{SmO}_{2}$ (thenar muscle) to indicate the need for blood transfusions in trauma patients; $\mathrm{SmO}_{2}$ values $<75 \%$ on admission were associated with the need for a transfusion. ${ }^{13}$ These characteristics make $\mathrm{SmO}_{2}$ an early and sensitive marker of central hypovolemia, with potential applications for the detection of hemorrhage.

Currently, $\mathrm{SmO}_{2}$ sensors are stand-alone monitors that can be used in research laboratories, and by the bedside in the clinical setting. Advances are required in the design and practical utility of these monitors, particularly in the development of "wearable" sensors for use on patients in the prehospital setting, or by individuals in high-risk professions such as soldiers on the battlefield, underground miners, and emergency first responders. Traditionally, the forearm and hand have been used as preferred anatomical sites to assess peripheral $\mathrm{SmO}_{2}$ during central hypovolemia. ${ }^{6,8} \mathrm{In}$ some circumstances, however, oxygen consumption in the arm and hand musculature may change simply due to increased movement of the arms, ${ }^{14}$ or peripheral limb sites may not be available due to traumatic amputation or other injury (e.g., burn). One potential step for improving "wearability" is moving sensors from the periphery to central locations such as the back.

We sought to evaluate the efficacy of three novel anatomical sites for the assessment of $\mathrm{SmO}_{2}$ under progressive central hypovolemia, including the deltoid (DELT) (upper arm), trapezius (TRAP) (neck/shoulder), and latissimus dorsi (LAT) (lower back). These sites were compared to the traditional site in the forearm (flexor carpi ulnaris muscle). The findings from this study could lead to improved detection and treatment of hemorrhagic injuries in numerous settings including emergency medicine, battlefield trauma, and the operating room.

\section{Materials and methods Subjects}

Ten healthy, non-smoking males participated in this study (age $25 \pm 2$ years; height $175 \pm 6 \mathrm{~cm}$; weight $77 \pm 14 \mathrm{~kg}$; mean $\pm \mathrm{SD}$ ) conducted at the University of North Texas Health Science Center (UNTHSC), Fort Worth, TX. All experimental procedures were conducted under a protocol approved by the UNTHSC Institutional Review Board. During a familiarization session, subjects provided written informed consent to participate in this study. Prior to inclusion, all subjects underwent a medical history evaluation, including a 12-lead ECG and orthostatic blood pressure assessment, and were cleared by a physician to participate. Subjects were instructed to abstain from caffeine, alcohol, dietary supplements, medications, and exercise for $24 \mathrm{~h}$ prior to the experiment, and to obtain a minimum of $6 \mathrm{~h}$ of sleep the night before testing.

\section{Study design}

LBNP was used to reduce central blood volume; this technique has recently been validated as an experimental method that simulates actual blood loss. ${ }^{15,16}$ With the use of a durable plastic sleeve and neoprene belt to form an airtight seal between the subject and the chamber, application of LBNP (below the iliac crest) results in a redistribution of blood volume away from the upper body (head and heart) to the abdomen and lower extremities.

Subjects were positioned in the LBNP chamber in the prone posture (i.e., face down) and were instrumented for measurement of HR via a standard lead II ECG (shielded leads, cable and amplifier, AD instruments, Bella Vista, NSW, Australia), and non-invasive arterial pressure via finger photoplethysmography (Finometer ${ }^{\mathrm{TM}}$, Finapres Medical Systems, Amsterdam, The Netherlands). Beat-to-beat, SV was also recorded directly from the Finometer, calculated by the pulse-contour method. ${ }^{17}$

$\mathrm{SmO}_{2}$ of the forearm (flexor carpi ulnaris; ARM), upper arm (DELT), and two sites on the back (LAT; TRAP) were obtained via NIRS (CareGuide ${ }^{\mathrm{TM}}$, Reflectance Medical Inc., Westborough, MA). The two arm sensors were placed inside a contoured disposable adhesive cover and positioned over each muscle. The two back sensors were placed directly over the muscle sites and taped securely in place. Heavy black cloth was placed over the sensors to block out any ambient light that could interfere with the NIRS signal. The absorption and scattering of multiple NIR wavelengths of light $(700-1000 \mathrm{~nm})$ were used for continuous calculation of oxy-hemoglobin $\left(\mathrm{HbO}_{2}\right)$ and deoxy-hemoglobin $(\mathrm{dHb})$ concentrations within the muscle for subsequent real-time calculation of $\mathrm{SmO}_{2}$ and muscle $\mathrm{pH}(\mathrm{pHm})$. All NIRS sensors were attached to the subject and collected spectra throughout the experimental set-up period (approximately 20-30 $\mathrm{min}$ ) to ensure stable signal acquisition prior to the commencement of the LBNP protocol.

The LBNP protocol consisted of a 5-min baseline period followed by progressive reductions in chamber pressure in 5-min intervals to $-15,-30,-45,-60,-70,-80,-90$, and $-100 \mathrm{mmHg}$. The protocol was terminated at the onset of hemodynamic decompensation or completion of $5 \mathrm{~min}$ at $-100 \mathrm{mmHg}$ LBNP. Hemodynamic decompensation was defined as one or a combination of the following criteria. (1) progressive diminution of systolic arterial pressure (SAP) $<80 \mathrm{mmHg}$; (2) sudden bradycardia, or (3) voluntary subject termination due to the onset of subjective presyncopal symptoms such as gray-out, nausea, sweating, dizziness, or general discomfort. Following LBNP termination, subjects remained in the LBNP chamber for a 10-min recovery period.

\section{Data analysis}

All continuous waveform data (ECG, arterial pressure, SV) were collected at $1000 \mathrm{~Hz}$ (PowerLab and Labchart, AD Instruments, Bella Vista, NSW, Australia) and analyzed offline via specialized software (WinCPRS, Absolute Aliens, Turku, Finland). R-waves generated from the ECG signal were detected and marked. HR was derived from the R-R interval signal. Diastolic arterial pressure (DAP) and SAP were marked from the arterial blood pressure tracings. Mean arterial pressure (MAP) was automatically calculated from the area under the arterial pressure tracing via the WinCPRS software. Cardiac output (CO) was calculated as the product of HR and SV, and TPR was calculated as MAP divided by CO. Muscle NIRS spectra were recorded 
every $30 \mathrm{sec}$ for each sensor. All variables were calculated from the final $3 \mathrm{~min}$ of baseline and each level of LBNP; the presyncopal values were calculated from the final $1 \mathrm{~min}$ prior to presyncope.

\section{Statistics}

One-way repeated measures ANOVAs were used for the assessment of all responses during LBNP, followed by Holm-Sidak post hoc tests to compare responses to baseline only. $\mathrm{SmO}_{2}$ and $\mathrm{pHm}$ measurements obtained at the ARM site were considered the established "standard" anatomical location; all statistical comparisons of the three novel anatomical sites were made with the ARM determinations. $\mathrm{SmO}_{2}$ and $\mathrm{pHm}$ responses during LBNP were compared between the ARM and each of the other three sites (DELT, LAT, and TRAP) via linear regression, and Pearson correlations (R-values), $P$-values, and slopes were determined for each comparison. The relationship between $\mathrm{SmO}_{2}$ responses at each site and SV was also compared via Pearson correlations. Comparison of these slopes between sites were made with either one-way ANOVAs or the non-parametric equivalent (Kruskal-Wallis test) if the data were not normally distributed (following multiple attempts at data transformation). The maximum change in $\mathrm{SmO}_{2}$ from baseline to presyncope was determined for each sensor site, and compared via one-way repeated measures ANOVA, followed by Holm-Sidak post hoc tests. Unless otherwise stated, all data are presented as mean \pm standard error (SE), and exact $P$ values are reported for all comparisons.

\section{Results}

Of the 10 subjects who completed this study, the LBNP protocol was terminated at $-70 \mathrm{mmHg}$ LBNP for five subjects, $-80 \mathrm{mmHg}$ for four subjects, and $-90 \mathrm{mmHg}$ for one subject. Data were analyzed up to $-80 \mathrm{mmHg}$ as at least five subjects made it to this level of LBNP. In response to progressive application of LBNP, SV decreased from $-15 \mathrm{mmHg}$ LBNP (Table 1), falling by a maximum of $46 \pm 6 \%$ at presyncope $(N=10)$. This reduction in central blood volume elicited an increase in HR by $-30 \mathrm{mmHg}$ LBNP, and a reduction in SAP by $-45 \mathrm{mmHg}$ LBNP. Decreases in $\mathrm{SmO}_{2}$ were detected at the ARM during the first level of LBNP $(-15 \mathrm{mmHg}$; Figure 1), the DELT at $-30 \mathrm{mmHg}$, the LAT at $-45 \mathrm{mmHg}$, and the TRAP at $-60 \mathrm{mmHg}$ LBNP. Decreases in $\mathrm{pHm}$ were also detected at the ARM site at $-15 \mathrm{mmHg}$ LBNP (Table 1), but not until $-45 \mathrm{mmHg}$ LBNP for the DELT, and $-60 \mathrm{mmHg}$ for both the LAT and TRAP sites.

$\mathrm{SmO}_{2}$ and $\mathrm{pHm}$ detected by all novel sensors tracked the $\mathrm{SmO}_{2}$ and $\mathrm{pHm}$ responses from the ARM sensor (Figure 2). The linear relationships (i.e., slope) were strongest for the LAT sensor compared with the TRAP $(P=0.03)$, and DELT $(P=0.08)$. The decreases in $\mathrm{SmO}_{2}$ detected by each sensor closely tracked the reduction in $\mathrm{SV}$, represented by high $\mathrm{R}$ values (Figure 3). Of the three novel sensors, the most linear relationship was observed at the LAT site, with the highest combined $\mathrm{R}$ value and slope. The ARM vs. SV slope was higher than the DELT $(P=0.003)$ and TRAP $(P=0.006)$ sites, and there were no differences between the LAT and DELT $(P=0.127)$, the LAT and TRAP $(P=0.210)$, or the ARM and LAT sites $(P=0.199)$.

When comparing the absolute change and percentage change in $\mathrm{SmO}_{2}$ from baseline to presyncope, there were no differences in these responses between the LAT and ARM sites $(P \geq 0.24)$, but the decreases detected by the TRAP $(P<0.001)$ and the DELT $(P=0.001)$ were smaller than the response at the ARM site (Table 2).

Table 1 Hemodynamic responses to central hypovolemia elicited by lower body negative pressure (LBNP) in the prone posture

\begin{tabular}{|c|c|c|c|c|c|c|c|}
\hline & \multicolumn{7}{|c|}{ LBNP level (mmHg) } \\
\hline & Baseline & -15 & -30 & -45 & -60 & -70 & -80 \\
\hline $\mathrm{HR}(\mathrm{bpm})$ & $65 \pm 2$ & $68 \pm 3$ & $73 \pm 3^{*}$ & $80 \pm 3^{*}$ & $90 \pm 3^{*}$ & $104 \pm 5^{\star}$ & $117 \pm 6^{\star}$ \\
\hline SAP $(\mathrm{mmHg})$ & $126.0 \pm 3.0$ & $126.1 \pm 3.0$ & $122.0 \pm 2.8$ & $118.7 \pm 3.1^{*}$ & $108.8 \pm 3.2^{*}$ & $103.8 \pm 2.4^{\star}$ & $95.1 \pm 3.2^{*}$ \\
\hline $\mathrm{DAP}(\mathrm{mmHg})$ & $74.4 \pm 1.4$ & $74.8 \pm 1.3$ & $74.7 \pm 1.4$ & $75.2 \pm 1.6$ & $71.7 \pm 1.5$ & $71.3 \pm 2.7$ & $67.8 \pm 2.4^{*}$ \\
\hline SV (ml) & $96.8 \pm 4.3$ & $90.1 \pm 3.8^{*}$ & $82.3 \pm 4.1^{*}$ & $72.7 \pm 3.9^{\star}$ & $62.8 \pm 4.4^{\star}$ & $50.3 \pm 3.7^{*}$ & $41.9 \pm 3.0^{\star}$ \\
\hline$\% \Delta \mathrm{SV}$ & - & $-6.8 \pm 1.2$ & $-14.8 \pm 2.6^{\star}$ & $-24.8 \pm 3.3^{*}$ & $-37.0 \pm 5^{\star}$ & $-48.3 \pm 3^{*}$ & $-56.9 \pm 2.3^{\star}$ \\
\hline $\mathrm{CO}(1 / \mathrm{min})$ & $6.4 \pm 0.3$ & $6.2 \pm 0.4$ & $6.0 \pm 0.4^{*}$ & $5.9 \pm 0.4^{\star}$ & $5.6 \pm 0.4^{\star}$ & $5.2 \pm 0.4^{\star}$ & $4.9 \pm 0.3^{*}$ \\
\hline TPR $\left(\mathrm{mmHg} / \mathrm{l} \cdot \mathrm{min}^{-1}\right)$ & $15.2 \pm 1.0$ & $15.6 \pm 1.0$ & $15.8 \pm 1.1$ & $16.0 \pm 1.1$ & $15.8 \pm 1.2$ & $16.1 \pm 0.9$ & $16.0 \pm 0.8$ \\
\hline $\mathrm{pHm}-\mathrm{ARM}$ & $7.42 \pm 0.02$ & $7.38 \pm 0.02^{*}$ & $7.35 \pm 0.02^{*}$ & $7.30 \pm 0.03^{\star}$ & $7.25 \pm 0.03^{*}$ & $7.20 \pm 0.06^{*}$ & $7.20 \pm 0.06^{\star}$ \\
\hline pHm-LAT & $7.46 \pm 0.01$ & $7.46 \pm 0.01$ & $7.45 \pm 0.01$ & $7.44 \pm 0.01$ & $7.42 \pm 0.02^{*}$ & $7.38 \pm 0.04^{*}$ & $7.36 \pm 0.04^{\star}$ \\
\hline pHm-TRAP & $7.39 \pm 0.05$ & $7.38 \pm 0.05$ & $7.38 \pm 0.05$ & $7.38 \pm 0.05$ & $7.37 \pm 0.05^{*}$ & $7.34 \pm 0.09^{*}$ & $7.33 \pm 0.09^{\star}$ \\
\hline pHm-DELT & $7.47 \pm 0.003$ & $7.46 \pm 0.003$ & $7.45 \pm 0.003$ & $7.45 \pm 0.005^{\star}$ & $7.44 \pm 0.01^{*}$ & $7.41 \pm 0.01^{*}$ & $7.41 \pm 0.01^{\star}$ \\
\hline
\end{tabular}

${ }^{*} P \leq 0.05$ vs. baseline.

Note: $N=10$ up to $-60 \mathrm{mmHg}$ LBNP; $N=5$ at -70 and $-80 \mathrm{mmHg}$ LBNP.

HR: heart rate; SAP: systolic arterial pressure; DAP: diastolic arterial pressure; MAP: mean arterial pressure; SV: stroke volume; CO: cardiac output; TPR: total peripheral resistance; ARM: flexor carpi ulnaris; LAT: latissimus dorsi; TRAP: trapezius; DELT: deltoid. 


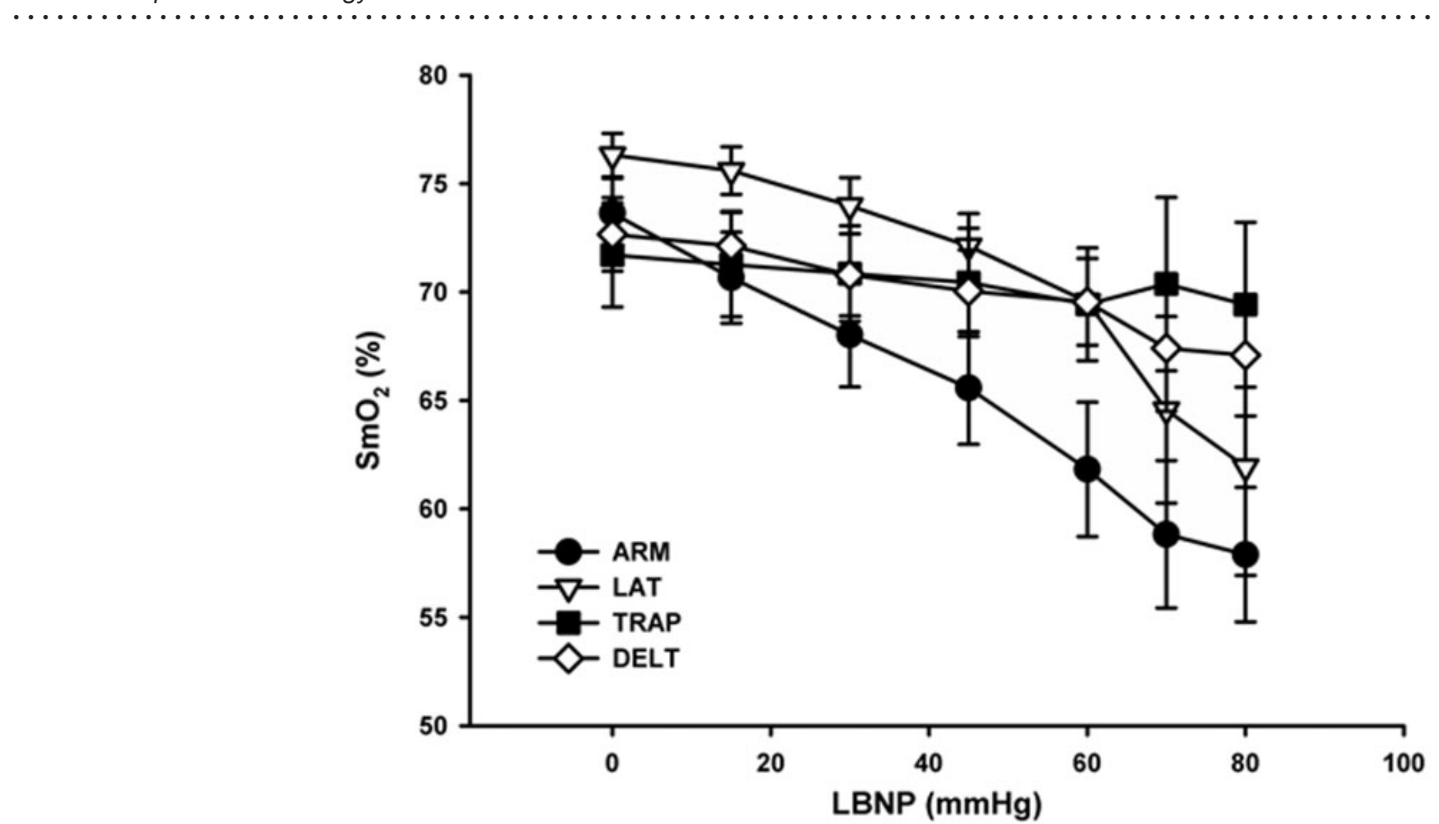

\begin{tabular}{|ccccccc|}
\hline LBNP Level & -15 & -30 & -45 & -60 & -70 & -80 \\
\hline $\mathbf{N}$ & 10 & 10 & 10 & 10 & 5 & 5 \\
\hline ARM P-Value & 0.007 & $<0.001$ & $<0.001$ & $<0.001$ & $<0.001$ & $<0.001$ \\
LAT P-Value & 0.568 & 0.129 & 0.005 & $<0.001$ & $<0.001$ & $<0.001$ \\
TRAP P-Value & 0.436 & 0.262 & 0.093 & 0.001 & $<0.001$ & $<0.001$ \\
DELT P-Value & 0.487 & 0.036 & $<0.004$ & $<0.001$ & $<0.001$ & $<0.001$ \\
\hline
\end{tabular}

Figure 1 Muscle tissue oxygen saturation $\left(\mathrm{SmO}_{2}\right)$ responses during central hypovolemia induced by lower body negative pressure (LBNP) at four sites, the flexor carpi ulnaris in the forearm (ARM), latissimus dorsi (LAT), trapezius (TRAP), and deltoid (DELT). Statistical comparison of responses during LBNP for each anatomical site compared with baseline at that site is presented in the table

\section{Discussion}

The findings of this investigation provide valuable insight into the potential utility of novel anatomical locations in detecting decreases in $\mathrm{SmO}_{2}$ during central hypovolemia (i.e., hemorrhage). The key findings are: (1) a progressive decrease in $\mathrm{SmO}_{2}$ was detected at all sites during central hypovolemia; (2) of the novel sites, the earliest reduction in $\mathrm{SmO}_{2}$ was observed at the DELT site, and the largest reduction in $\mathrm{SmO}_{2}$ was observed in the LAT site, and; (3) of the novel sites, the LAT site exhibited the most linear relationship with the reduction in central blood volume (i.e., SV). It appears that NIRS-derived $\mathrm{SmO}_{2}$ at the novel sites investigated in the current study can adequately track decreases in central blood volume, albeit with differing sensitivity. As the LAT sensor $\mathrm{SmO}_{2}$ responses exhibited the strongest linear relationships to the ARM sensor $\mathrm{SmO}_{2}$, and to the reduction in $\mathrm{SV}$, we conclude that the novel LAT sensor shows the highest efficacy for tracking central blood volume loss during simulated hemorrhage.

Of the novel sensor sites, the DELT $\mathrm{SmO}_{2}$ was reduced at the earliest level of central hypovolemia (i.e., $-30 \mathrm{mmHg}$ LBNP), when SV was reduced by $\sim 15 \%$. In comparison, $\mathrm{SmO}_{2}$ at the more distal ARM site was reduced below baseline from the first level of LBNP when SV was reduced by $\sim 7 \%$. The finding that decreases in $\mathrm{SmO}_{2}$ were detected at the two arm sites prior to the central sites may be due to preferential peripheral vasoconstriction elicited with central hypovolemic stress; further experimental studies are required to elucidate this finding (e.g., using vascular ultrasound to detect changes in vascular caliber at multiple, progressively more peripheral sites), and the underling mechanism (e.g., transduction of sympathetic activity, alpha-adrenergic receptor density and/or sensitivity, sympatholysis). In support of this proposal, however, is the finding that the correlation between TPR and $\mathrm{SmO}_{2}$ was higher for the ARM $(R=0.86, P=0.01)$ and DELT $(R=$ $0.86, P=0.01)$ sites compared with the more central sites ( $\mathrm{R}=0.77, P=0.05$ for LAT; $\mathrm{R}=0.72, P=0.07$ for TRAP). The delayed reduction in $\mathrm{SmO}_{2}$ at the DELT versus more distal ARM site suggests, however, that this vasoconstrictive effect (via the vascular arm of the baroreflex) does not occur simultaneously across all peripheral vascular beds. The observation that the reductions in muscle $\mathrm{pH}$ tracked the reductions in $\mathrm{SmO}_{2}$ for each sensor is likely due to decreased oxygen availability resulting from decreased flow. With a decrease in oxygen delivery, glycolysis results in a greater accumulation of $\mathrm{H}^{+}$within the muscle, and thus a greater reduction in $\mathrm{pH}$. A caveat, however, is that 


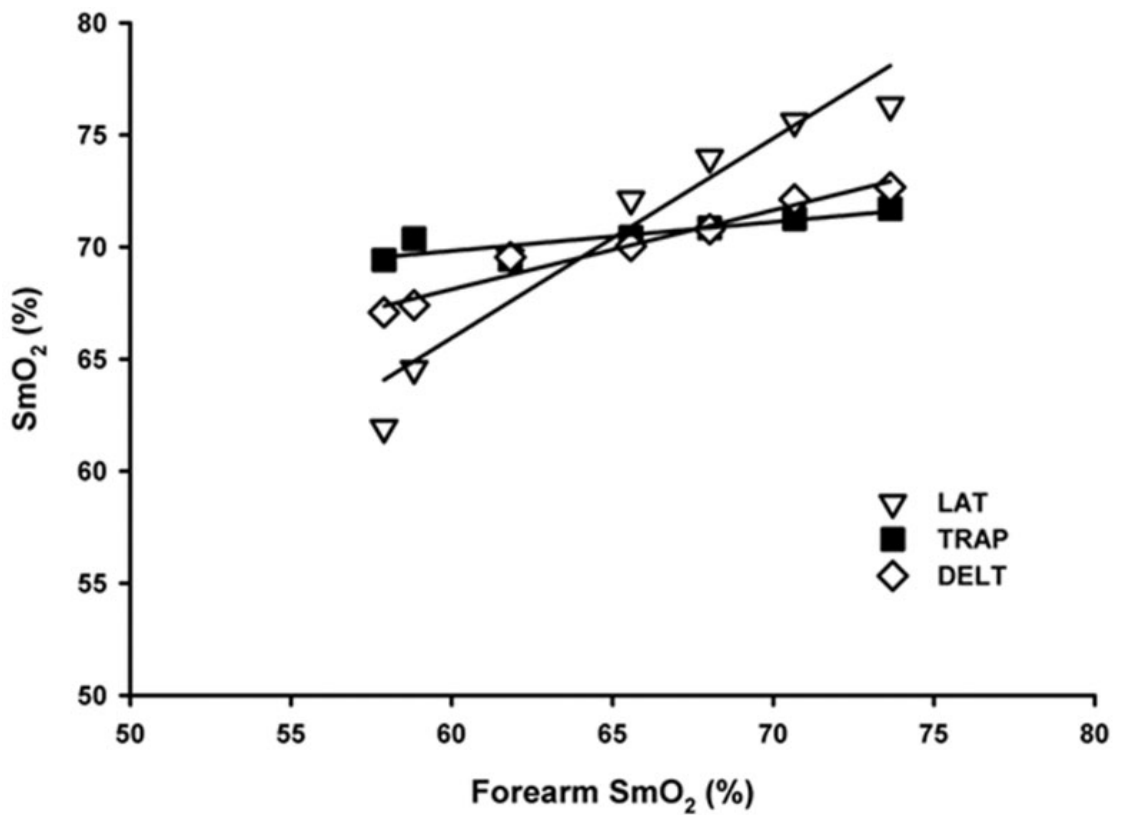

\begin{tabular}{|ccccccc|}
\hline & \multicolumn{3}{c}{$\mathrm{SmO}_{2}$ ARM } & \multicolumn{3}{c|}{ pH ARM } \\
\hline & $\mathrm{R}$ & P-value & Slope & $\mathrm{R}$ & P-value & Slope \\
\hline LAT & 0.96 & $<0.001$ & 0.89 & 0.93 & 0.002 & 0.41 \\
TRAP & 0.89 & 0.007 & 0.13 & 0.93 & 0.002 & 0.22 \\
DELT & 0.98 & $<0.001$ & 0.35 & 0.95 & $<0.001$ & 0.25 \\
\hline
\end{tabular}

Figure 2 Relationship between muscle tissue oxygen saturation $\left(\mathrm{SmO}_{2}\right)$ at three novel sites vs. forearm $\mathrm{SmO}$ during central hypovolemia induced by lower body negative pressure. R-values, $P$-values, and slopes for each comparison are presented in the table. LAT: latissimus dorsi; TRAP: trapezius; DELT: deltoid

this interpretation is based on the assumption that there was no change in oxygen extraction, which was not directly measured in this investigation. As our subjects were in a supine, sedentary posture, however, we have no reason to suspect that oxygen demand was altered at these muscle sites throughout the experiment, so it is unlikely that oxygen extraction increased.

Unexpectedly, $\mathrm{SmO}_{2}$ derived from the central LAT site tracked both the $\mathrm{SmO}_{2}$ responses of the peripheral ARM site, and the reduction in SV. LAT $\mathrm{SmO}_{2}$ was reduced below baseline when SV was reduced by $\sim 25 \%$. While the LAT is a centrally located muscle, which may infer blood flow protection under hypovolemic stress, non-active skeletal muscle is a not an essential organ under these conditions, with blood flow preferentially redistributed to the vital organs of the heart and brain. Advantages of utilizing centrally located muscle sites for the assessment of $\mathrm{SmO}_{2}$ include the absence of limb movement, which may in itself elicit reductions in $\mathrm{SmO}_{2},{ }^{14}$ and the potential for insertion of monitors into clothing as a "wearable sensor," for use in the military and emergency first responder settings.

While the present investigation supports the use of novel anatomical sites for the assessment of $\mathrm{SmO}_{2}$ during simulated hemorrhage, future work should seek to determine if these measurements are valid during actual bleeding injuries, and in combination with other potentially confounding factors, such as during increased activity or exercise, and pain. For example, if an individual is in an active state immediately prior to a hemorrhagic insult (such as during combat), or a patient is moving the muscle site where the sensor is placed, increased muscle activity will also affect $\mathrm{SmO}_{2}{ }^{18,19}$ even in the absence of decreasing central blood volume. Ideally, injury detection algorithms should be developed that can allow differentiation between decreases in $\mathrm{SmO}_{2}$ that occur with increased muscle activity (e.g., with movement sensors), and those that are characteristic of blood loss. A further practical consideration for use of these sensors in trauma is the potential influence of pain, which could affect the $\mathrm{SmO}_{2}$ responses via increased sympathetic nerve activity. One recent study demonstrated that $2 \mathrm{~min}$ of pain elicited by submerging the hand in ice water (i.e., the cold pressor test, known to reliably increase sympathetic nerve activity) reduced $\mathrm{SmO}_{2}$ in the forearm; this response was augmented when superimposed with $4 \mathrm{~min}$ of central hypovolemia via LBNP to $-60 \mathrm{mmHg}$. ${ }^{11}$

A limitation of the current investigation was that the hypovolemic stimulus and subsequent $\mathrm{SmO}_{2}$ measurements were only assessed during normothermia. A recent 


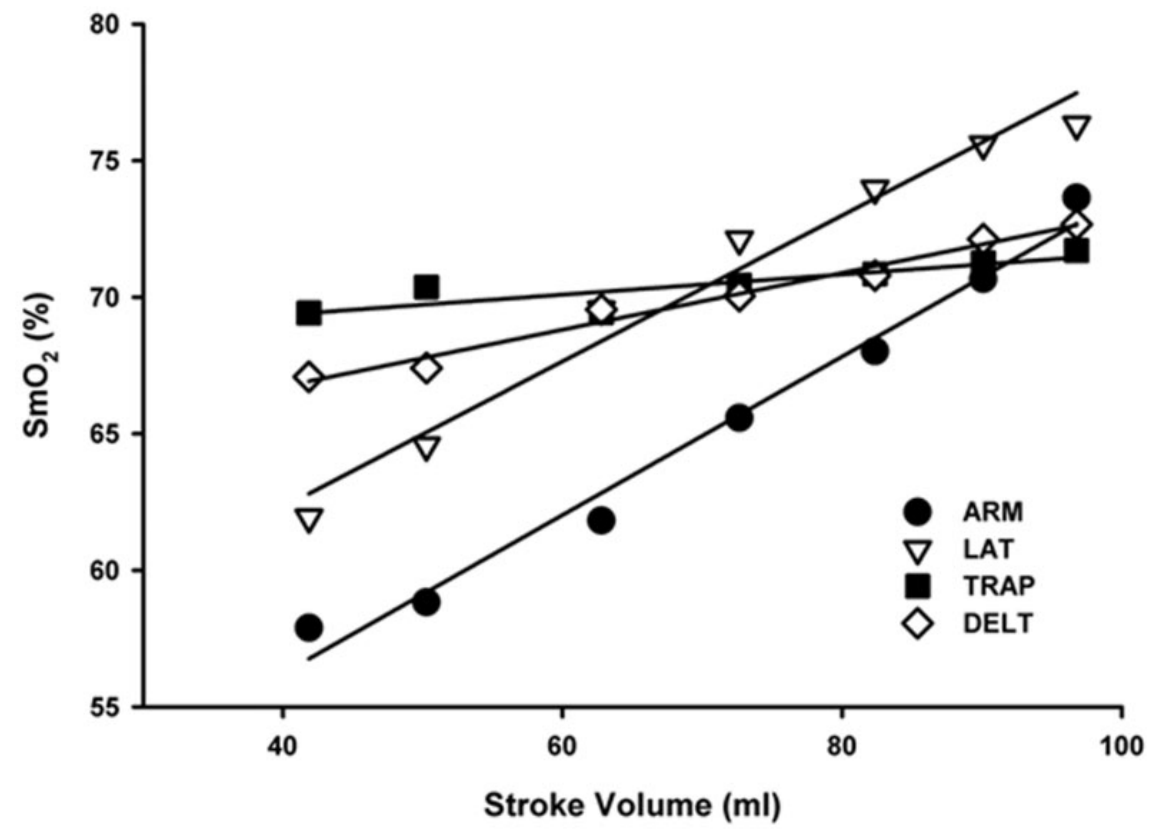

\begin{tabular}{|cccc|}
\hline \multicolumn{4}{c|}{$\mathrm{SmO}_{2}$ vs. Stroke Volume } \\
\hline & $\mathrm{R}$ & P-value & Slope \\
\hline ARM & 0.99 & $<0.001$ & 0.29 \\
LAT & 0.99 & $<0.001$ & 0.27 \\
TRAP & 0.87 & 0.01 & 0.04 \\
DELT & 0.99 & $<0.001$ & 0.10 \\
\hline
\end{tabular}

Figure 3 Relationship between muscle tissue oxygen saturation $\left(\mathrm{SmO}_{2}\right)$ at four sites and stroke volume during central hypovolemia induced by lower body negative pressure. R-values, $P$-values, and slopes for each comparison are presented in the table. ARM: flexor carpi ulnaris; LAT: latissimus dorsi; TRAP: trapezius; DELT: deltoid

Table 2 Absolute and percent change in muscle tissue oxygen saturation $\left(\mathrm{SmO}_{2}\right)$ from baseline to presyncope during progressive stepwise application of lower body negative pressure (LBNP)

\begin{tabular}{llclc}
\hline & $\begin{array}{l}\Delta \mathrm{SmO}_{2} \text { from } \\
\text { baseline } \\
\text { to presyncope }\end{array}$ & $\begin{array}{l}\boldsymbol{P} \text {-value } \\
\text { vs. ARM }\end{array}$ & $\begin{array}{l}\% \Delta \mathrm{SmO}_{2} \text { from } \\
\text { baseline } \\
\text { to presyncope }\end{array}$ & $\begin{array}{l}\boldsymbol{P} \text {-value } \\
\text { vs. ARM }\end{array}$ \\
\hline ARM & $-12.5 \pm 2.3$ & - & $-17.2 \pm 3.1$ & - \\
LAT & $-10.0 \pm 2.7$ & 0.24 & $-13.3 \pm 3.6$ & 0.19 \\
TRAP & $-3.0 \pm 1.1$ & $<0.001$ & $-4.2 \pm 1.6$ & $<0.001$ \\
DELT & $-4.5 \pm 1.4$ & 0.001 & $-6.1 \pm 2.1$ & 0.001 \\
\hline
\end{tabular}

ARM: flexor carpi ulnaris; LAT: latissimus dorsi; TRAP: trapezius; DELT: deltoid.

study from Schlader et al. ${ }^{20}$ suggests that $\mathrm{SmO}_{2}$ responses during central hypovolemia under hyperthermic conditions are smaller compared with the normothermic condition, presumably due to hyperthermia-induced increases in skin blood flow. This issue is particularly relevant to the application of these monitors in austere environments, such as the military setting, underground mines, and emergency first responders, where heat stress (environmental and situational) is a common occupational hazard. Assessment of potential contamination of $\mathrm{SmO}_{2}$ from hyperthermia-induced increases in skin blood flow at all anatomical sites is warranted.

Additionally, as the present investigation only considered male subjects, future work should assess whether these findings can be generalized to females. A recent study by Ellerby et al. ${ }^{21}$ assessed the CareGuide sensors at several anatomical sites (calf, DELT, and thigh) and found no effect of sex or age on the $\mathrm{SmO}_{2}$ values under resting conditions. ${ }^{21}$ Future studies should confirm these responses under hypovolemic stress.

Finally, for the purposes of the present study, subjects were placed in the prone position to optimize access to the two novel anatomical sites on the back. From a practical standpoint, however, patients are routinely placed on their back for treatment and transport, so assessment of muscle oxygenation responses while these sites are compressed should be conducted to ensure accurate and consistent responses with the findings of the present investigation.

In conclusion, while peripheral muscle sites such as the forearm and hand remain the "gold standard" locations for 
bedside monitoring of $\mathrm{SmO}_{2}$, all three novel locations investigated in the current study would enable early detection and tracking of the progressive decrease in central blood volume. Of the three novel sites, however, the LAT shows the most promise for utilization in the clinical setting.

Author contributions: CAR and BRS are responsible for conception and design of the work; CAR and JDS conducted experiments; JDS, BRS, and CAR analyzed data; JDS and CAR prepared figures; JDS drafted manuscript; JDS, BRS, and CAR edited and revised the manuscript critically for important intellectual content, and approved the final version to be published.

\section{ACKNOWLEDGEMENTS}

The authors would like to thank our subjects for their time and cheerful cooperation, and Drs. Albert Yurvati and Levi Rice for their assistance with subject medical examinations. Funding for this study was provided via a contract with Reflectance Medical Inc.

\section{DECLARATION OF CONFLICTING INTERESTS}

At the time of the study, BRS was an employee and officer of Reflectance Medical Inc., and holds stock and stock options in the company.

\section{REFERENCES}

1. Brown CV, Velmahos GC, Neville AL, Rhee P, Salim A, Sangthong B, Demetriades D. Hemodynamically "stable" patients with peritonitis after penetrating abdominal trauma: identifying those who are bleeding. Arch Surg 2005;140:767-72

2. Cocchi MN, Kimlin E, Walsh M, Donnino MW. Identification and resuscitation of the trauma patient in shock. Emerg Med Clin North Am 2007;25:623-42

3. Convertino VA, Ryan KL, Rickards CA, Salinas J, McManus JG, Cooke WH, Holcomb JB. Physiological and medical monitoring for en route care of combat casualties. J Trauma 2008;64(4 Suppl): S342-53

4. Adams SL, Greene JS. Absence of a tachycardic response to intraperitoneal hemorrhage. J Emerg Med 1986;4:383-9

5. Victorino GP, Battistella FD, Wisner DH. Does tachycardia correlate with hypotension after trauma? J Am Coll Surg 2003;196:679-84

6. Bartels SA, Bezemer R, de Vries FJ, Milstein DM, Lima A, Cherpanath TG, van den Meiracker AH, van Bommel J, Heger M, Karemaker JM, Ince C. Multi-site and multi-depth near-infrared spectroscopy in a model of simulated (central) hypovolemia: lower body negative pressure. Intensive Care Med 2011;37:671-7

7. Lee J, Kim JG, Mahon S, Tromberg BJ, Ryan KL, Convertino VA, Rickards CA, Osann K, Brenner M. Tissue hemoglobin monitoring of progressive central hypovolemia in humans using broadband diffuse optical spectroscopy. J Biomed Opt 2008;13:064027

8. Soller BR, Ryan KL, Rickards CA, Cooke WH, Yang Y, Soyemi OO, Crookes BA, Heard SO, Convertino VA. Oxygen saturation determined from deep muscle, not thenar tissue, is an early indicator of central hypovolemia in humans. Crit Care Med 2008;36:176-82

9. Soller BR, Yang Y, Soyemi OO, Ryan KL, Rickards CA, Walz JM, Heard SO, Convertino VA. Noninvasively determined muscle oxygen saturation is an early indicator of central hypovolemia in humans. J Appl Physiol 2008;104:475-81

10. Soller BR, Zou F, Ryan KL, Rickards CA, Ward K, Convertino VA. Lightweight noninvasive trauma monitor for early indication of central hypovolemia and tissue acidosis: a review. J Trauma Acute Care Surg 2012;73(2 Suppl 1): S106-11

11. Hoiseth LO, Hisdal J, Hoff IE, Hagen OA, Landsverk SA, Kirkeboen KA Tissue oxygen saturation and finger perfusion index in central hypovolemia: influence of pain. Crit Care Med 2015;43:747-56

12. Ward KR, Tiba MH, Ryan KL, Filho IP, Rickards CA, Witten T, Soller BR, Ludwig DA, Convertino VA. Oxygen transport characterization of a human model of progressive hemorrhage. Resuscitation 2010;81:987-93

13. Carlile C, Wade CE, Baraniuk MS, Holcomb JB, Moore LJ. Evaluation of $\mathrm{StO} 2$ tissue perfusion monitoring as a tool to predict the need for lifesaving interventions in trauma patients. Am J Surg 2015;210:1070-5

14. Soller BR, Yang Y, Lee SM, Wilson C, Hagan RD. Noninvasive determination of exercise-induced hydrodgen ion threshold through direct optical measurement. J Appl Physiol 2008;104:837-44

15. Hinojosa-Laborde C, Shade RE, Muniz GW, Bauer C, Goei KA, Pidcoke HF, Chung KK, Cap AP, Convertino VA. Validation of lower body negative pressure as an experimental model of hemorrhage. J Appl Physiol 2014;116:406-15

16. Johnson BD, van Helmond N, Curry TB, van Buskirk CM, Convertino VA, Joyner MJ. Reductions in central venous pressure by lower body negative pressure or blood loss elicit similar hemodynamic responses. J Appl Physiol 2014;117:131-41

17. Jansen JR, Wesseling KH, Settels JJ, Schreuder JJ. Continuous cardiac output monitoring by pulse contour during cardiac surgery. Eur Heart J 1990 December;(11 Suppl I):26-32

18. Belardinelli R, Barstow TJ, Porszasz J, Wasserman K. Changes in skeletal muscle oxygenation during incremental exercise measured with near infrared spectroscopy. Eur J Appl Physiol Occup Physiol 1995;70:487-92

19. Lee SM, Clarke MS, O'Connor DP, Stroud L, Ellerby GE, Soller BR. Near infrared spectroscopy-derived interstitial hydrogen ion concentration and tissue oxygen saturation during ambulation. Eur J Appl Physiol 2011;111:1705-14

20. Schlader ZJ, Rivas E, Soller BR, Convertino VA, Crandall CG. Tissue oxygen saturation during hyperthermic progressive central hypovolemia. Am J Physiol Regul Integr Comp Physiol 2014;307:R731-6

21. Ellerby GE, Smith CP, Zou F, Scott P, Soller BR. Validation of a spectroscopic sensor for the continuous, noninvasive measurement of muscle oxygen saturation and pH. Physiol Meas 201;34:859-71

(Received April 21, 2016, Accepted June 27, 2016) 